\title{
Characterising the SCUBA-2 superconducting bolometer arrays
}

\author{
Dan Bintley $^{1 \mathrm{a}}$, Michael J. MacIntosh ${ }^{\mathrm{b}}$, Wayne S. Holland ${ }^{\mathrm{b}, \mathrm{d}}$, Per Friberg ${ }^{\mathrm{a}}$, Craig Walther ${ }^{\mathrm{a}}$, David \\ Atkinson $^{\mathrm{b}}$, Dennis Kelly ${ }^{\mathrm{b}}$, Xiaofeng Gao ${ }^{\mathrm{b}}$, Peter A.R. Ade ${ }^{\mathrm{c}}$, William Grainger ${ }^{\mathrm{c}}$, Julian House ${ }^{\mathrm{c}}$, \\ Lorenzo Moncelsi ${ }^{\mathrm{c}}$, Matthew I. Hollister, ${ }^{\mathrm{d}, \mathrm{i}}$, Adam Woodcraft ${ }^{\mathrm{d}}$, Camelia Dunare ${ }^{\mathrm{e}}$, William Parkes ${ }^{\mathrm{e}}$, \\ Anthony J. Walton ${ }^{\mathrm{e}}$, Kent D. Irwin ${ }^{\mathrm{f}}$, Gene C. Hilton ${ }^{\mathrm{f}}$, Michael Niemack ${ }^{\mathrm{f}}$, Carl D. Reintsema ${ }^{\mathrm{f}}$, \\ Mandana Amiri ${ }^{\mathrm{g}}$, Bryce Burger ${ }^{\mathrm{g}}$, Mark Halpern ${ }^{\mathrm{g}}$, Matthew Hasselfield ${ }^{\mathrm{g}}$, Jeff Hill ${ }^{\mathrm{h}}$, J. B. Kycia ${ }^{\mathrm{h}}$, \\ C.G.A. Mugford ${ }^{\text {h }}$, Lauren Persaud ${ }^{\text {h }}$ \\ a Joint Astronomy Centre, 660 N. A'ohoku Place, Hilo, HI 96720 \\ ${ }^{b}$ UK Astronomy Technology Centre, Royal Observatory, Blackford Hill, Edinburgh EH9 3HJ, UK

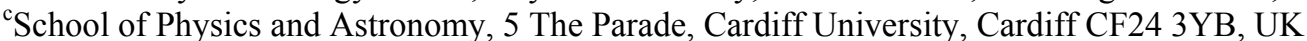 \\ ${ }^{\mathrm{d} I n s t i t u t e}$ for Astronomy, University of Edinburgh, Royal Observatory, Blackford Hill, Edinburgh EH9 3HJ, UK \\ ${ }^{\mathrm{e}} \mathrm{S}$ cottish Microelectronics Centre, University of Edinburgh, West Mains Road, Edinburgh EH9 3JF, UK \\ ${ }^{\mathrm{f}}$ National Institute of Standards and Technology, 325 Broadway, Boulder, CO 80305 \\ ${ }^{g}$ Department of Physics and Astronomy, University of British Columbia, British Columbia V6T 1Z1, Canada \\ ${ }^{\mathrm{h}}$ Department of Physics, University of Waterloo, Waterloo, Ontario, N2L 3G1, Canada \\ ${ }^{\mathrm{i}}$ California Institute of Technology, Pasadena, CA 91125
}

\begin{abstract}
SCUBA-2 is a state of the art 10,000 pixel submillimeter camera installed and being commissioned at the James Clerk Maxwell Telescope (JCMT) providing wide-field simultaneous imaging at wavelengths of 450 and 850 microns. At each wavelength there are four 32 by 40 sub-arrays of superconducting Transition Edge Sensor (TES) bolometers, each packaged with inline SQUID multiplexed readout and amplifier. In this paper we present the results of characterising individual 1280 bolometer science grade sub-arrays, both in a dedicated 50mk dilution refrigerator test facility and in the instrument installed at the JCMT.
\end{abstract}

Keywords: TES, transition edge sensor, low temperature detector, SQUID, SCUBA-2, submillimeter astronomy

\section{INTRODUCTION}

SCUBA-2 is a new wide-field common user camera, being commissioned at the James Clerk Maxwell Telescope (JCMT) on the summit of Mauna Kea, Hawai'i. Operating simultaneously at two wavelengths $(450 \mu \mathrm{m}$ and $850 \mu \mathrm{m})$, with a 50 sq-arcmin field of view, SCUBA-2 is set to revolutionise submillimeter astronomy. SCUBA-2 will be an unprecedented imaging and survey instrument, capable of mapping large areas of sky to great depth (100 times faster than the original SCUBA instrument it replaces), addressing key questions about the origins of galaxies, stars and planets.

The new instrument and re-imaging mirrors were mounted and aligned on the JCMT to schedule, a major engineering success. At 3.5 tonnes and with dimensions of $2.3 \mathrm{~m} \mathrm{x} 2 \mathrm{~m} \times 2 \mathrm{~m}$, SCUBA-2 is by far the largest instrument in the history of JCMT. The engineering challenges and achievements of SCUBA-2 in this regard are described in these proceedings by Craig et al. ${ }^{1}$

SCUBA-2 came to Hawai'i with two commissioning grade detector sub-arrays with known flaws and limitations, but of sufficient quality to begin the instrument commissioning. Fully populated the two focal planes will each consist of four 1280 Transition Edge Sensor (TES) bolometer sub-arrays. Early commissioning highlighted two problems that would undermine the performance of future science grade arrays in the instrument: Sensitivity to the motion of the antenna (and

${ }^{1}$ d.bintley@jach.hawaii.edu; phone (808) 961-3756; fax (808) 961-6516

Millimeter, Submillimeter, and Far-Infrared Detectors and Instrumentation for Astronomy V, edited by Wayne S. Holland, Jonas Zmuidzinas, Proc. of SPIE Vol. 7741, 774106

(c) 2010 SPIE $\cdot$ CCC code: $0277-786 X / 10 / \$ 18 \cdot$ doi: $10.1117 / 12.856839$

Proc. of SPIE Vol. $7741774106-1$ 
hence the instrument) rotating in azimuth and the $450 \mu \mathrm{m}$ detectors saturated (went normal) on the sky. Both problems have been successfully addressed. Firstly the magnetic shielding on the focal plane units was improved by using a custom single piece welded niobium shield, rather than a number of overlapping niobium panels. Secondly the optical loading on the $450 \mu \mathrm{m}$ focal plane was reduced by narrowing the $450 \mu \mathrm{m}$ pass band filter profile and by eliminating the potential for reflections from the inside of the focal plane covers (done for both wavelengths).

In early 2009, the first pair (one of each wavelength) of science quality sub-arrays were completed and tested in a dedicated dilution fridge facility at Cardiff University, UK, before being installed in SCUBA-2 at JCMT. On sky commissioning and a short science observing campaign have followed. The first science results are very promising and will soon be published. The remaining six sub-arrays have now been completed and will be installed in the instrument later this summer (July 2010). The original pair of science grade sub-arrays have also been upgraded with new cold electronics modules (CEM), to correct an adverse thermal loading from $1 \mathrm{~K}$, discussed below.

In this paper we focus on the dark testing and characterisation of the first pair of science grade sub-array modules both in the Cardiff test facility and installed in SCUBA-2 on the JCMT. Elsewhere in these proceedings Holland et al. ${ }^{2}$ provide a full update on the SCUBA-2 project, including the first astronomical results, while Dempsey et al. ${ }^{3}$ discuss the on-sky calibration of the instrument.

\section{SCUBA-2 DETECTORS}

\subsection{Sub-array modules}

Individual sub-arrays are packaged as standalone low temperature modules, connected to their own dedicated MCE (Multi Channel Electronics; room temperature control and readout electronics). This modular approach allows for flexibility. We have been able to test and commission the instrument with various configurations of prototype sub-arrays installed, while detector and MUX development, testing and fabrication have continued in parallel; on occasion with simultaneous cold testing in Edinburgh, Waterloo and Cardiff.

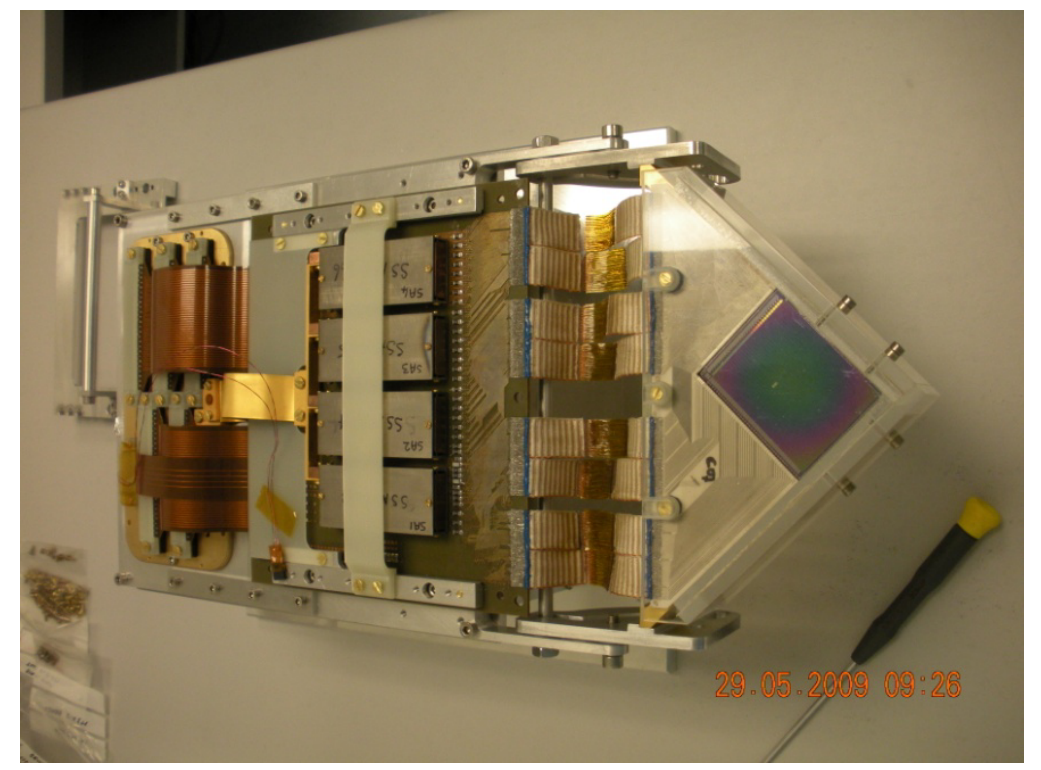

Figure 1. SCUBA-2 science grade sub-array module in a handling jig, ready for unfolding.

The detector array and SQUID multiplexer are fabricated separately on two (actually three, as the detector wafer is two wafers bonded together to provide mechanical support during processing) silicon wafers before joining or hybridising with indium bump bonds. ${ }^{4}$ The bump bonds provide both thermal and electrical contact between the two layers. The upper detector wafer contains 1280 DC coupled molybdenum-copper TES detectors, in a 40 row, 32 column format. A silicon nitride membrane provides a weak thermal link to the thermal bath. 
Each detector pixel includes a resistive heater used to compensate for changes in optical power as the sky background changes, enabling detectors to be operated at a chosen bias point for a wide range of sky powers. The heaters play a fundamental role in the operation of the instrument while observing:

- Detectors are individually calibrated by measuring their responsivity using a 'fast heater ramp', (a triangle wave on the heater of a few $\mathrm{pW}$, peak-peak) at the start of and if we choose, periodically during every observation.

- The optical power from the sky is directly measured by using heater tracking. A servo loop on the heater is run to keep detector output constant while opening and closing the cold shutter from dark to the sky. Periodic heater tracking transfers the slow changes in sky power to the heater setting, keeping each detector close to the original setup operating point in the superconducting transition.

The incorporation of the heater into the design of the detector device and use of the heater while observing is one of the innovative features of SCUBA-2.

The $450 \mu \mathrm{m}$ and $850 \mu \mathrm{m}$ detectors have a similar physical design for ease of manufacture, (the $850 \mu \mathrm{m}$ focal plane fully samples an area of sky, whereas the $450 \mu \mathrm{m}$ focal plane is under-sampled by a factor of 2). The transition temperature of the $450 \mu \mathrm{m}$ TES is made higher, to allow for greater power handling and a relaxed NEP requirement. The functional requirement at both wavelengths is that the instrument is sky background noise limited on the telescope.

The lower wafer is a NIST designed and fabricated time division SQUID multiplexer (MUX) with 32 channels, each with 41 rows. The 41 st row is a 'dark row' without any TES element. The dark row SQUID output is available to the data reduction pipeline, to remove excess noise particularly at low frequencies and magnetic pickup. It is the SQUID MUX which makes large-scale TES arrays such as SCUBA-2 practical, by vastly reducing the wire count between the detectors and the room temperature electronics. The operation and design of the time division multiplexer for SCUBA-2 is described by de Korte et al. ${ }^{5}$
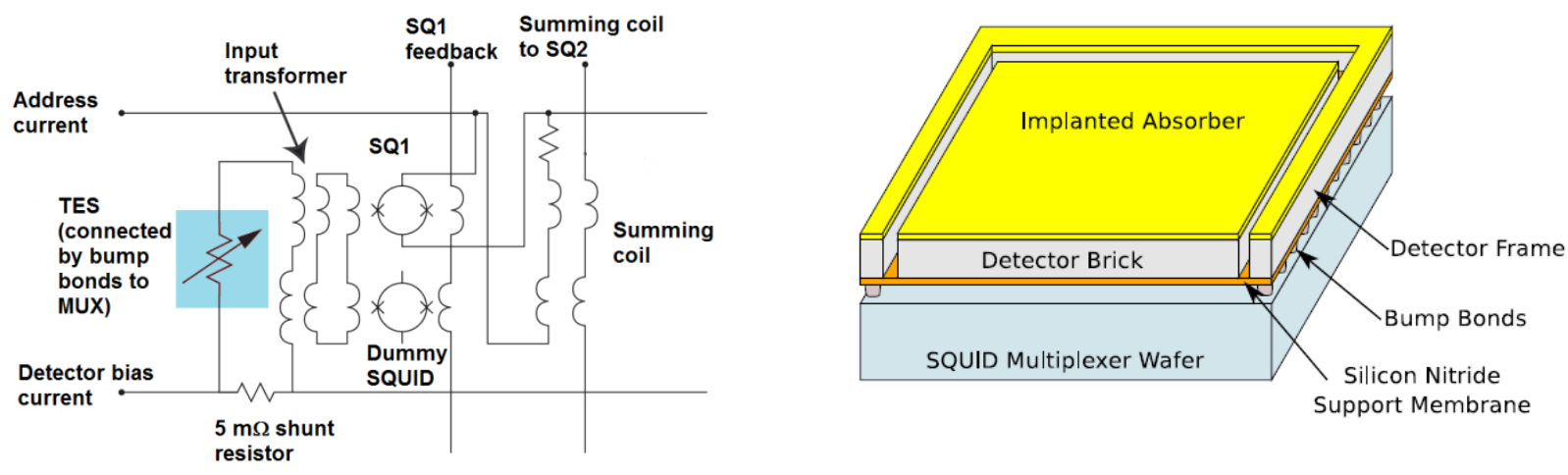

Figure 2. The circuit diagram and schematic drawing of a single TES/MUX element. The electrical and thermal contacts between the detector wafer and the SQUID MUX are made by cold welded indium bump bonds.

On the MUX wafer a first stage SQUID (SQ1) sits below each TES detector element. The coupling between the TES and the SQ1 is by magnetic flux. Current flowing through the TES generates a magnetic field at the SQ1 via an input transformer on the MUX wafer (see Figure 2). Each channel or column of 41 SQ1s is then coupled by a summing coil to the second stage SQUID (SQ2). The signals in each channel are amplified by a 100 SQUID Series Array (SSA) located on the $1 \mathrm{~K}$ PCB of the cold electronics module (CEM).

The SCUBA-2 TES detectors are operated in an approximate voltage biased mode, using a small $5 \mathrm{~m} \Omega$ shunt resistor located on the MUX wafer. The operation of Transition Edge Sensors in this fashion is described by Irwin ${ }^{6}$ and Irwin and Hilton ${ }^{7}$, where they summarise the TES small signal theory, applicable to the SCUBA-2 detectors.

The advantage of voltage biasing the TES, is that negative electro-thermal feedback (ETF) stabilises the detector against thermal runaway. An increase in background power warms the device and causes an increase in resistance. This in turn causes the detector current to decrease, cooling the TES. Strong ETF essentially keeps the temperature of the TES constant, while providing a simple and direct relation between any applied power (optical or heater) and the current 
flowing through the device. Negative feedback also makes the detector self-biasing in terms of temperature in the transition. This is important for large arrays of TES such as with SCUBA-2. If a variation of transition temperatures and transition widths exists across the array and they do not all overlap, the Joule power dissipation in each TES will cause it to self heat to within its respective superconducting transition. Negative feedback can also increase the bandwidth of electrical systems including TES. Changes in the incident power are compensated for by changes in the bias current power on timescales shorter than the thermal time constant of the detector.

The current flowing through each TES is measured by its SQ1. The output of a SQUID is periodic with magnetic flux from the input coil; the periodicity being given by a flux quantum. There is no unique output for a given detector current. Therefore we use the first stage SQUID as a null detector. Feedback current is applied by the room temperature electronics (the MCE) to the SQ1 feedback coil to null the field from the TES detector current in the input coil. By applying a flux locked loop (FLL), the applied feedback current is proportional to the current flowing through the TES. We refer to measurements using the flux locked loop as 'closed loop' measurements. In open loop measurements we take the output directly from the SQ1, with no feedback applied.

The dynamic range of the detector feedback circuit is limited by the available first stage SQUID feedback current and the mutual inductance of the SQ1 input coil. These parameters have been carefully chosen to meet the stringent noise requirements of the instrument. However using the MCE we have been able to increase the dynamic range by flux jumping. The SQUID response is periodic and the period is measured as part of the sub-array set-up. It is possible for the MCE to jump or slip multiple flux quanta, while keeping count of the number jumped and remain locked in the FLL. The dynamic range is increased seamlessly, towards the physical limits of the TES power handling. With too much applied power (optical, thermal, bias) the TES becomes normal and ceases to work as a bolometer, too little and the TES becomes superconducting, to the same effect.

\subsection{Upgrading to Science Grade Arrays}

The manufacture of the SCUBA-2 sub-array is a long process with many steps and testing at each stage. Prior to hybridisation, MUX wafers, which are by far the most complex of the component parts, are individually characterised in a dedicated $4 \mathrm{~K}$ facility at the University of Waterloo. Testing at this stage allows minor fabrication faults to be identified and corrected. The expected detector yield, based on the uniformity of the first stage SQUID properties is calculated for each MUX wafer.
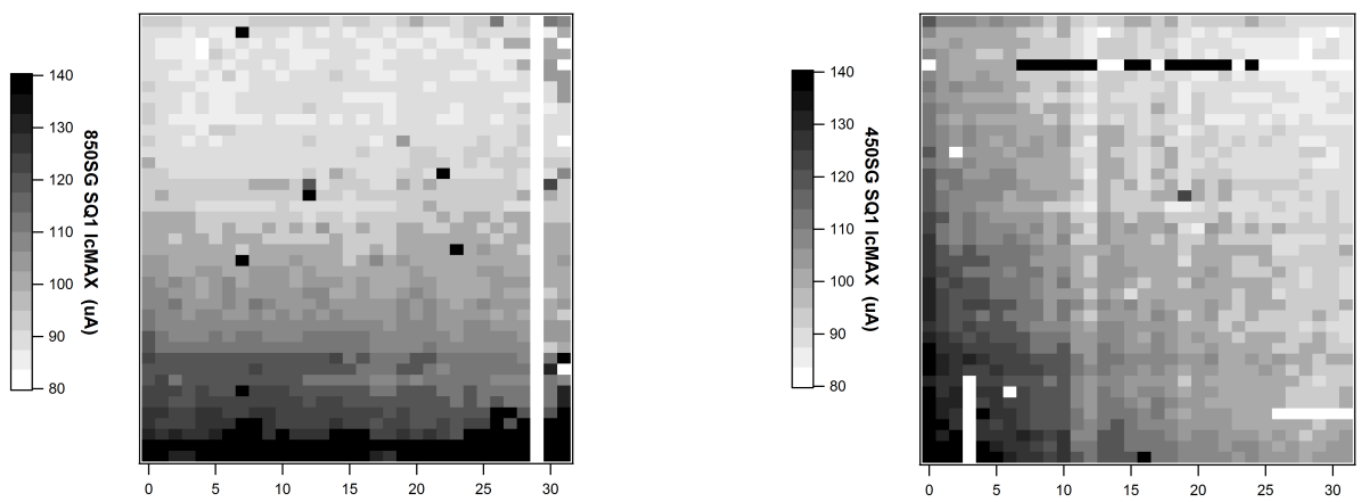

Figure 3. The results of SQ1 Icmax measurements, done in a dedicated 4K facility, at the University of Waterloo: (Left) $850 \mu \mathrm{m}$ science grade MUX: (Right) $450 \mu \mathrm{m}$ MUX.

The time based multiplexing scheme used requires a common SQ1 bias applied per row, together with a single value of SQ2 feedback per column. When setting up of the sub-arrays, these two parameters are optimised to maximise the number of working detectors. By using the same optimisation algorithm on the Waterloo $4 \mathrm{~K}$ screening data, we can predict the expected detector yield for a particular MUX. For the latest generation of multiplexer yields exceed $95 \%$. An example of the MUX screening results are given in Figure 3: We show the SQ1 Icmax values, which relate to the SQUID bias currents that give maximum modulation of the V- $\phi$ characteristic, for the MUX wafers used for the first science 
grade arrays. The gradients in optimal SQ1 bias are an artefact of the fabrication process. They make setting up the MUX with a single SQ1 bias per row and a single SQ2 feedback current per column more complex.

The detector wafers are carefully matched to the MUX wafers to ensure reliable bump bonding. The bilayer TES, heater and associated 'wiring' are fabricated on the underside of the prepared detector wafer; the transition temperature is tuned by adjusting the thickness of the metal layers. Witness samples are produced during the process, which are then cooled to mk temperatures to verify the TES transition temperature of each wafer.

The bump bonding process is not without risk. We discovered during the processing of prototype arrays, that a vital cleaning step will aggressively eat away the metal from the TES devices if any pinholes in the protective layer are present, changing the properties of the TES. The $850 \mu \mathrm{m}$ commissioning array was processed without the cleaning step. Unfortunately we found that this left the central region of detectors unstable with different characteristics to the edges. This was attributed to the formation of an oxide layer between the two halves of some of the indium bump bonds. At the edges of the wafer the detectors worked as expected. Here the bonding force might have broken through the oxide layer producing a contiguous superconducting bond. The solution for the science grade arrays was to apply the cleaning step, but only after careful and laborious inspection of the detector wafer for pin holes.

Following hybridisation, the upper detector wafer is deep-etch micro-machined to reveal the individual detectors standing on a silicon nitride membrane. The finished detector/MUX is glued to a beryllium copper 'hairbrush' thermal and mechanical support mounting block and then wire bonded into the cold electronics module. Room temperature electrical checks and visual inspections are done after each step in the process.

The design of the cold electronics module has proved very challenging. Over 300 electrical connections are required between the MUX wafer at $60 \mathrm{mk}$ and a PCB at $1 \mathrm{~K}$. The original solution was to use custom made superconducting, niobium tracks on kapton flexes, to link the $1 \mathrm{~K}$ PCB and a ceramic board (batwing) with silver plated copper tracks thermally attached to the hairbrush mount. Aluminium wedge wire bond links are made from the silver pads on the ceramic board to the MUX.

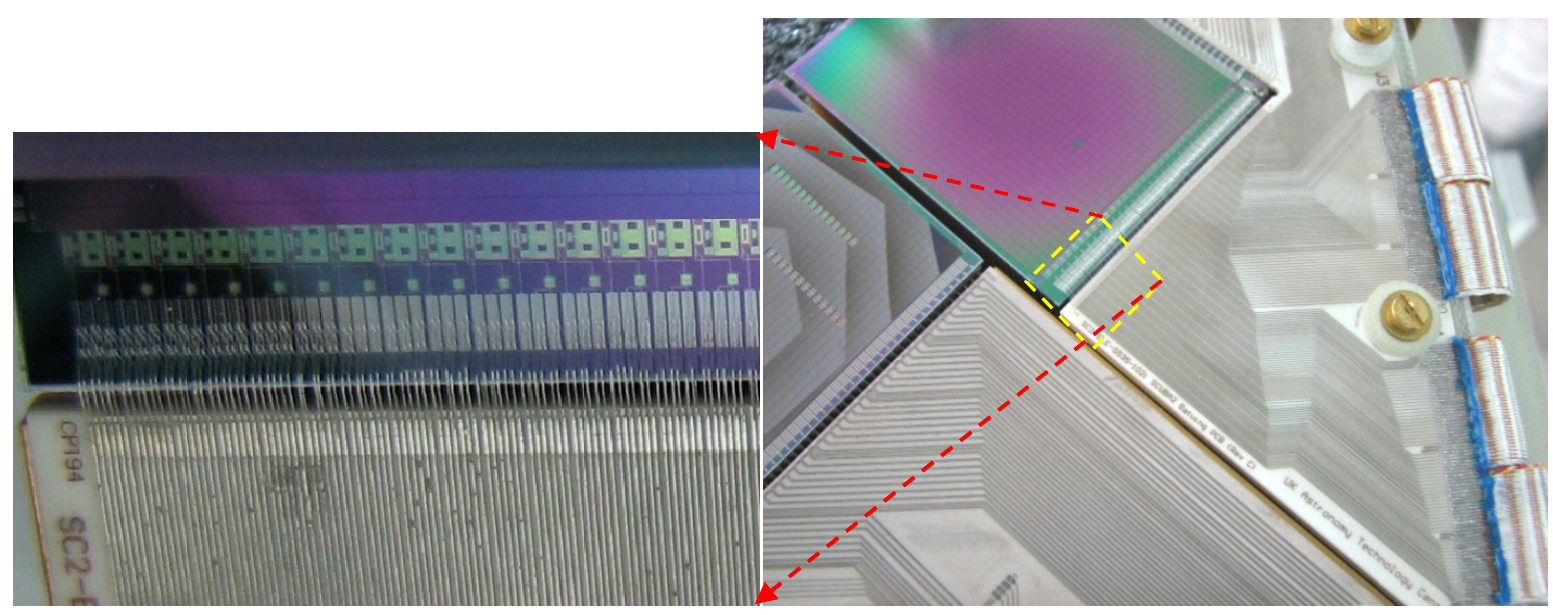

Figure 4. Science grade array with the new style cold electronics module, showing the soldered connections to the batwing. In the expanded view, the wire bonding, second stage SQ2s and dark row SQ1s are visible.

Testing of the prototype and commissioning arrays revealed a weakness in the wire bonding process and a possible heating effect in some of the kapton flex to batwing joints. In some cases the heating was sufficient to prevent the subarray from operating. We switched off those channels on prototype sub-arrays that caused heating, to allow the remainder of the array to function.

The cold electronics module was redesigned for the science grade sub-arrays: The kapton flexes were replaced with niobium-titanium wire directly soldered to pads on the ceramic batwing and after many wire bond thermal cycling and pull tests, the wire bond problem has now been understood and largely eliminated. However testing of the science grade arrays revealed a new problem. The new NbTi wiring introduced an excessive heat flow from $1 \mathrm{~K}$ to the sub-arrays and the fridge mixing chamber. The NbTi wires are copper clad then insulated. The copper adds strength and allows the wire 
to be easily soldered. To create thermal isolation, the copper was removed from each wire for a length in the centre of the cable. The wires were then reinsulated with GE varnish, to prevent electrical shorts.

A test module for new design CEM successfully cooled down to $60 \mathrm{mk}$ (at the cold end) in the Cardiff system. But as explained below; with the $850 \mu \mathrm{m}$ science grade array installed in the Cardiff system, the fridge base temperature was held at over $100 \mathrm{mk}$ compared to the usual $50 \mathrm{mk}$. The problem was traced to the variability of the application of GE varnish. The GE varnish thickness was reduced for the $450 \mu \mathrm{m}$ sub-array, which reduced the heat leak but did not eliminate the problem. The $850 \mu \mathrm{m}$ was then repackaged to match the $450 \mu \mathrm{m}$ before shipping to Hawai'i.

A further redesign of the CEM has been undertaken. The cladding on the niobium-titanium wire is changed to phosphor bronze, which provides the required thermal performance without the need for a break. The new wire woven into ribbon cable has been extensively tested at low temperature at the UKATC and by the low temperature group in Florence. This new CEM will be used for the new batch of science grade arrays (including upgrading the original pair).

\section{SUB-ARRAY TESTING}

The initial two science grade sub-arrays were tested individually, in a dedicated dilution refrigerator facility at the University of Cardiff, before careful transport and integration into SCUBA-2 at the JCMT. The final batch of detector arrays will be integrated directly into the Instrument without further cold testing.

The Cardiff test bed has been used to test a number of prototype SCUBA-2 sub-arrays. The magnetic shielding is similar to that on the instrument, as is the arrangement of light tight cold shields surrounding the detector array. The test bed has no window to the Lab (although the design is such that one could be incorporated); instead a cryogenic black body fills the field of view of the detector array. The black body together with three point source fast switching SPIRE type calibrated illuminators are mounted inside the $1 \mathrm{~K}$ shield to facilitate optical testing. The installed filters have similar characteristics to those in the instrument. The dilution fridge is conventional in that there is a liquid He bath and a pumped $1 \mathrm{~K}$ pot to condense the returning mixture. However the cryostat is modified for optical testing. It has no IVC, so heat switches instead of exchange gas are used for the initial cool down. It is instructive to compare detector performance in the Cardiff system to that in SCUBA-2, to check for evidence of the pulse tubes, shutter motor, light leaks etc in the instrument affecting the performance of the detectors.
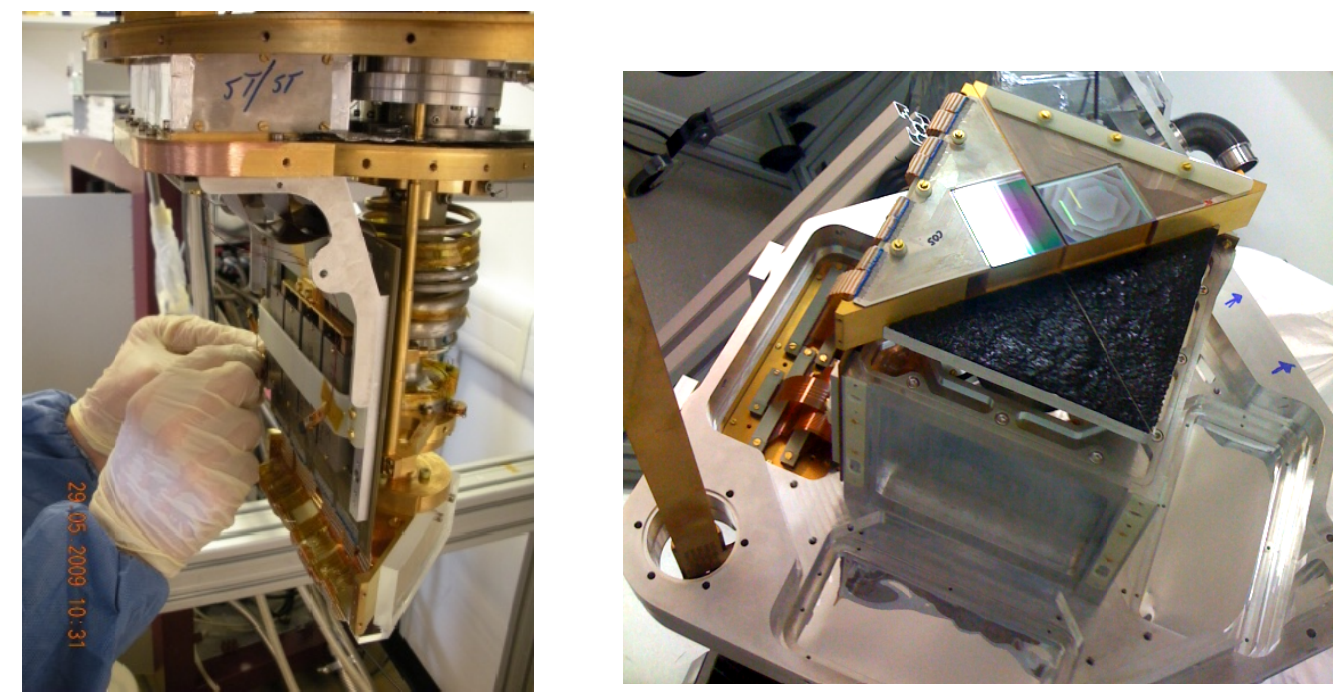

Figure 5. (Left) SCUBA-2 sub-arrays installed in the Cardiff dilution fridge. The hairbrush support is bolted directly to the mixing chamber, while the cold electronics are cooled by the still. (Right) Sub-arrays installed in the SCUBA-2 focal plane unit (FPU) at JCMT. 
Previous tests on prototype arrays in the Cardiff test bed have been reported by Woodcraft et al. ${ }^{8}$ The most significant recent progress in testing sub-arrays has been due to the advances in the use of the MCE. Rather than just characterising individual detectors, we are able to characterise the whole array. This is important because there are many compromises that have to be made to setup an array into the best state for observing. Not every detector can be biased into the optimum part of the transition. Compromises are also made with SQUID setup, which directly impacts the performance of individual bolometers.

In previous papers ${ }^{9}$ we have described the software used for the automatic SQUID setup process. We have successfully automated the process to optimally adjust the 3000 or so parameters that control the MUX operation for the whole subarray in less than 3 minutes. A faster setup, which only adjusts the SSA and SQ2 feedback currents and optimises the SQ1 bias, is used during observing on the telescope. The setup software continues to evolve as we acquire more experience.

The quality of the SQUID setup directly affects the performance of the detectors. We observed lower noise from the system with the SSA and SQ2 biased to give maximum V- $\phi$ modulation. For the SQ1, we aim to use the minimum bias after optimisation, which has each SQ1 in a row fully on (which tends to coincide with maximum modulation). The bandwidth of the MUX with the SQUIDs biased in this way has been measured using the MCE in the $50 \mathrm{MHz}$ fast mode and it is found to be sufficient.

The setup procedure itself can be run from the command line or within a script. Scripts can manipulate TES bias or TES heater, taking measurements after each change to simulate applying signals to either line. The instrument fridge temperature is also controllable from the command line or within a script, as is the cold shutter position and external blackbody source. The majority of the measurements to characterise a TES array have been automated in this way.

\subsection{Array Characterisation, Basic Tools}

Using the $\mathrm{MCE}^{10}$, the ability to automate measurement of the TES array is limited mainly by the imagination of the user. At the heart of the MCE are a number of FPGAs. If a particular measurement is not possible with the standard MCE firmware, the FPGA can be reprogrammed to make it so.

One example of the MCE flexibility is a fast mode that allows measurements to be made at the $50 \mathrm{MHz}$ MCE clock speed. Normally this would not be possible due to limits in the maximum data throughput out of the MCE. In fast mode the MCE stores the measurements, which are then read out of the buffer using the standard MCE command. With fast mode it is possible to investigate the MUX switching and settling times to optimise the multiplexing frame rate and other FLL timing parameters. Another example is an MCE generated heater or TES bias square wave function, used to measure detector time constants.

However the most useful and commonly used measurement scripts are quite simple:

- ramp_bias: This is a script which generates a small triangle wave on the TES bias or heater.

- ramp_bias_vi: This script ramps the TES bias over a (user selected) much larger range, to generate IV characteristics. Usually the ramp is from the normal state into the transition.

- mceframetest: Collects a time stream of SQ1 feedback current (or direct output if open loop) from all detectors in the array at a selected frame rate (up to a few $\mathrm{kHz}$, with the firmware version we use). By combining a measurement of responsivity (ramp_bias using the heater) with a current noise measurement, the NEP of each detector is measured.

A slightly more sophisticated script is used to measure each detector's dynamic thermal conductance $(\mathrm{G}=\mathrm{dP} / \mathrm{dT})$ and superconducting transition temperature, using an open loop, ramping heater method. The script simultaneously measures the heater power required to put each individual detector at the top or normal end of the superconducting transition with minimal TES bias applied. This is repeated for a range of different temperatures.

We operate the MCE in two modes; engineering mode for setup and array characterisation and science mode for observing. However the distinction between the modes is reducing, as many of the tasks that previously required engineering mode, can now be done in science mode. The fundamental difference between the modes is that in science mode the MCEs are fully integrated into the telescope control and observing system. The output from the multiple MCEs 
are controlled by an external sync box which supplies a clock signal and requests data frames at $200 \mathrm{~Hz}$. The data is subsequently packed into the standard starlink NDF format, which contain extensive header information for pipeline processing. In engineering mode, multiple MCE can also be run from the external sync box clock, however the control of the MCE is independent of the telescope system and data is saved in a simple text based data frame format with headers added by the control scripts.

The clock rate of the MCE (from internal or external clock) is $50 \mathrm{MHz}$. We currently operate with a multiplexing frame rate of $9.53 \mathrm{kHz}$. That is we multiplex 41 rows of SQ1s and stay on each row for $12850 \mathrm{MHz}$ clock cycles. After a settling period, a calculated SQ1 feedback is applied based on the previous frame measurement and then after a further waiting period, the output sampled and co-added for a further number of clock cycles. The SQ1 bias for that row is then turned off and the next energised and so on. The MCE has various data modes. We most commonly return filtered SQ1 feedback. In filtered mode, the MCE applies a digital 4-pole Butterworth low-pass filter to the SQ1 feedback for output, matched to the $200 \mathrm{~Hz}$ science mode data output frame rate. The MCE filter response can be seen in figure 13 .

Using the four scripts described above, the properties of individual TES detectors or the whole sub-array can be mapped out for different TES bias, heater and temperature setting. There are many interlinked properties that determine the successful operation of a TES array. However for the operation of SCUBA-2 on the telescope, the key performance figures are the detector NEP and NEFD (noise equivalent flux density). These properties have some distribution for the detectors in each focal plane. The modal value and distribution of the NEP and NEFD directly affect the mapping speed of the instrument.

\subsection{Additional heat load from the science grade sub-arrays}

The $850 \mu \mathrm{m}$ science grade sub-array was cooled down first in the Cardiff system. The fridge base temperature was much higher than expected. We estimated that the sub-array was contributing an additional $36 \mu \mathrm{W}$ heat load on the mixing chamber, compared to previous prototype sub-arrays modules. Thermal modelling indicated that a thick layer of GE varnish used to insulate the bare niobium-titanium wires in the thermal breaks was the likely cause. The $450 \mu \mathrm{m}$ science grade had a thinner layer of GE varnish. With the $450 \mu \mathrm{m}$ sub-array in the system, the fridge base temperature was close to $50 \mathrm{mk}$ but still elevated. Following testing in Cardiff the $850 \mu \mathrm{m}$ CEM was changed to match the $450 \mu \mathrm{m}$. Installed in SCUBA-2, the instrument base temperature is similarly elevated and the MUX temperatures of both science grade subarrays are $15 \mathrm{mk}$ warmer than the installed commissioning grade arrays. The higher MUX temperature has a significant affect on performance, particularly for the $850 \mu \mathrm{m}$ sub-array.

\section{$3.3850 \mu \mathrm{m}$ Tc Flattening}
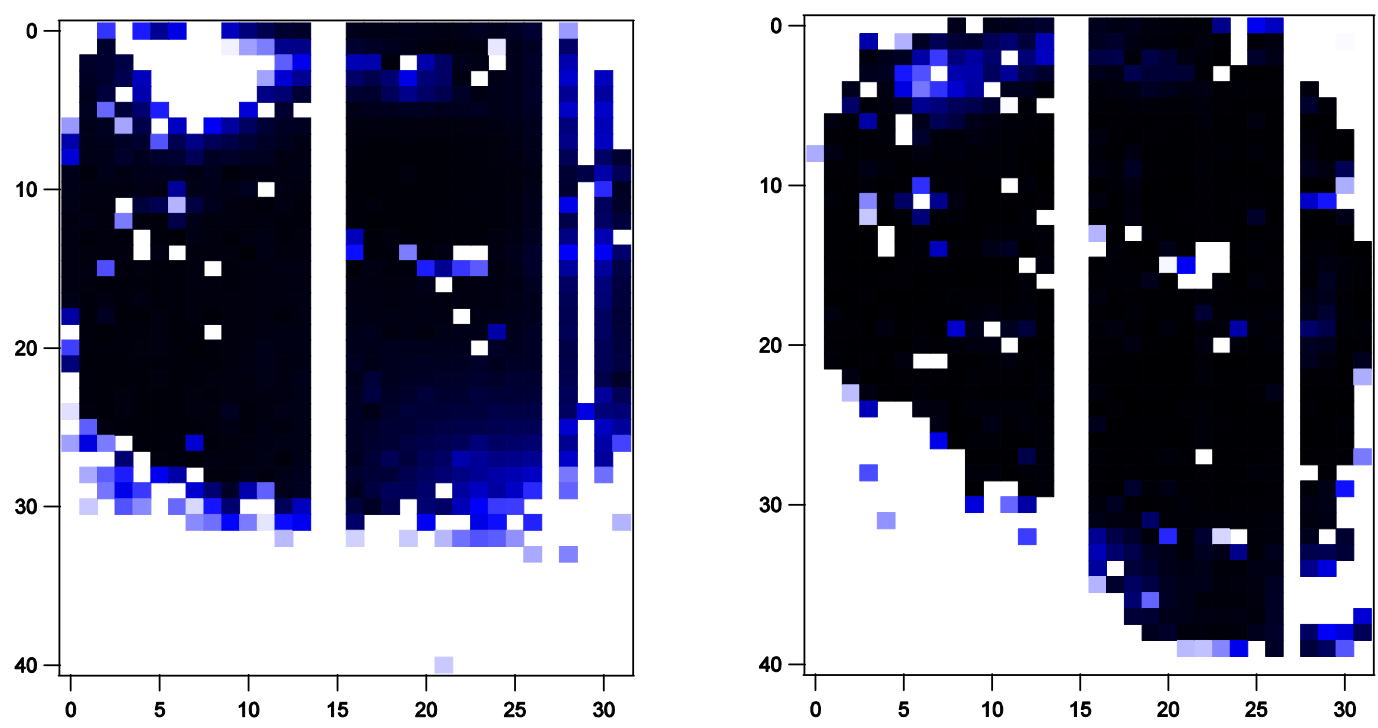

Figure 6. The results of Tc flattening on the $850 \mu \mathrm{m}$ sub-array; (Left) no flattening, (right) with flattening. The SQ1 bias current is used to selectively apply heat to the detectors, to smooth out the Tc variation. 
The science grade $850 \mu \mathrm{m}$ detectors have a large spread in measured Tc, perhaps exaggerated by the excess heat leak from $1 \mathrm{~K}$ causing uneven heating of the MUX wafer. This is a significant factor for setting up this sub-array. To smooth out the effect of the variation in Tc, a novel technique of 'Tc flattening' has been developed. By applying a higher SQ1 bias on selected number of rows for a few clock cycles at the start of the 128 cycles each row is on, the SQ1 are used as heaters. Tc flattening is row based, so it cannot correct for a Tc variation across the columns. For the $850 \mu \mathrm{m}$ we are able to recover 10 or so rows (although not every column in the row), which would not otherwise have been in the transition, with no apparent adverse effects. The results of Tc flattening are shown in Figure 6.

\subsection{Science grade sub-array detector characteristics and performance}

We describe the measured characteristics of typical science grade 450 and $850 \mu \mathrm{m}$ TES detectors.

Table 1. Measured characteristics for science grade arrays.

\begin{tabular}{|l|l|l|}
\hline & $\mathbf{4 5 0 \mu m}[\mathrm{r} 16 \mathrm{c} 3]$ & $\mathbf{8 5 0 \mu m}[\mathrm{r} 10 \mathrm{c} 20]$ \\
\hline Normal State Resistance & $59.7 \mathrm{~m} \Omega$ & $28.2 \mathrm{~m} \Omega$ \\
\hline Total Power (Heater $+\mathbf{~ I V})$ & $274 \mathrm{pW}$ & $43.2 \mathrm{pW}$ \\
\hline Transition Temperature & $194.8 \mathrm{mk}$ & $135 \mathrm{mk}$ \\
\hline Thermal conductance G (= dP/dT) & $6.0 \mathrm{nW} / \mathrm{K}$ & $1.8 \mathrm{nW} / \mathrm{K}$ \\
\hline Phonon limited NEP & $1.12 \times 10^{-16} \mathrm{~W} / \sqrt{ } \mathrm{Hz}$ & $4.2 \times 10^{-17} \mathrm{~W} / \sqrt{ } \mathrm{Hz}$ \\
\hline
\end{tabular}
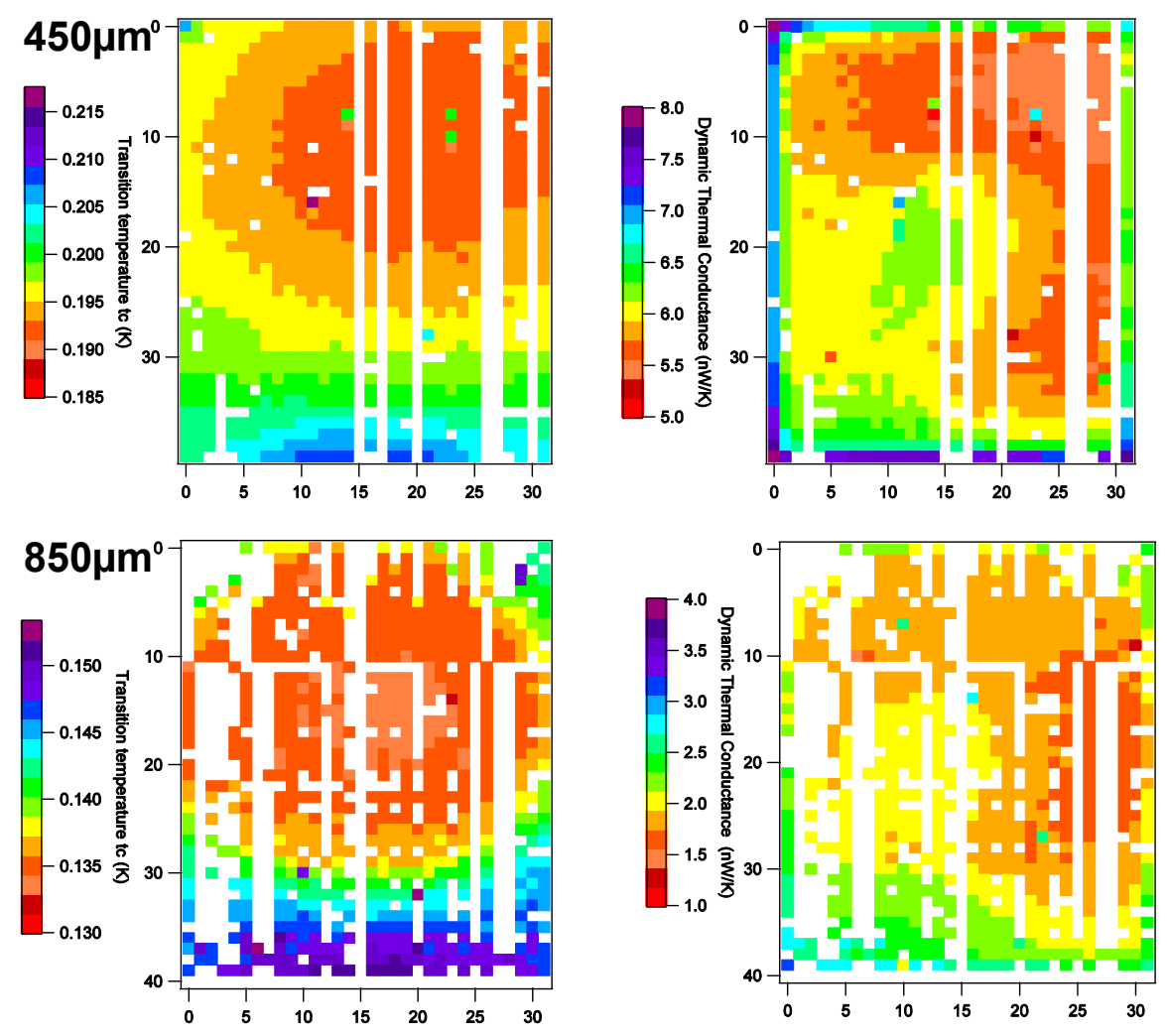

Figure 7. Measured transition temperatures (left) and thermal conductance (right), for the $450 \mu \mathrm{m}$ and $850 \mu \mathrm{m}$ science grade subarrays. 
Transition temperatures and thermal conductance are shown in Figure 7. From these measurements the intrinsic phonon noise limited NEP $=\sqrt{4 K_{B} T^{2} G}$, can be estimated (assuming a $1 \mathrm{~Hz}$ bandwidth). The phonon noise limited NEP for the $450 \mu \mathrm{m}$ detectors are shown in Figure 8, together with a comparison of the phonon noise limited NEP and NEP measured from the detector current noise in the dark for the $850 \mu \mathrm{m}$ array.
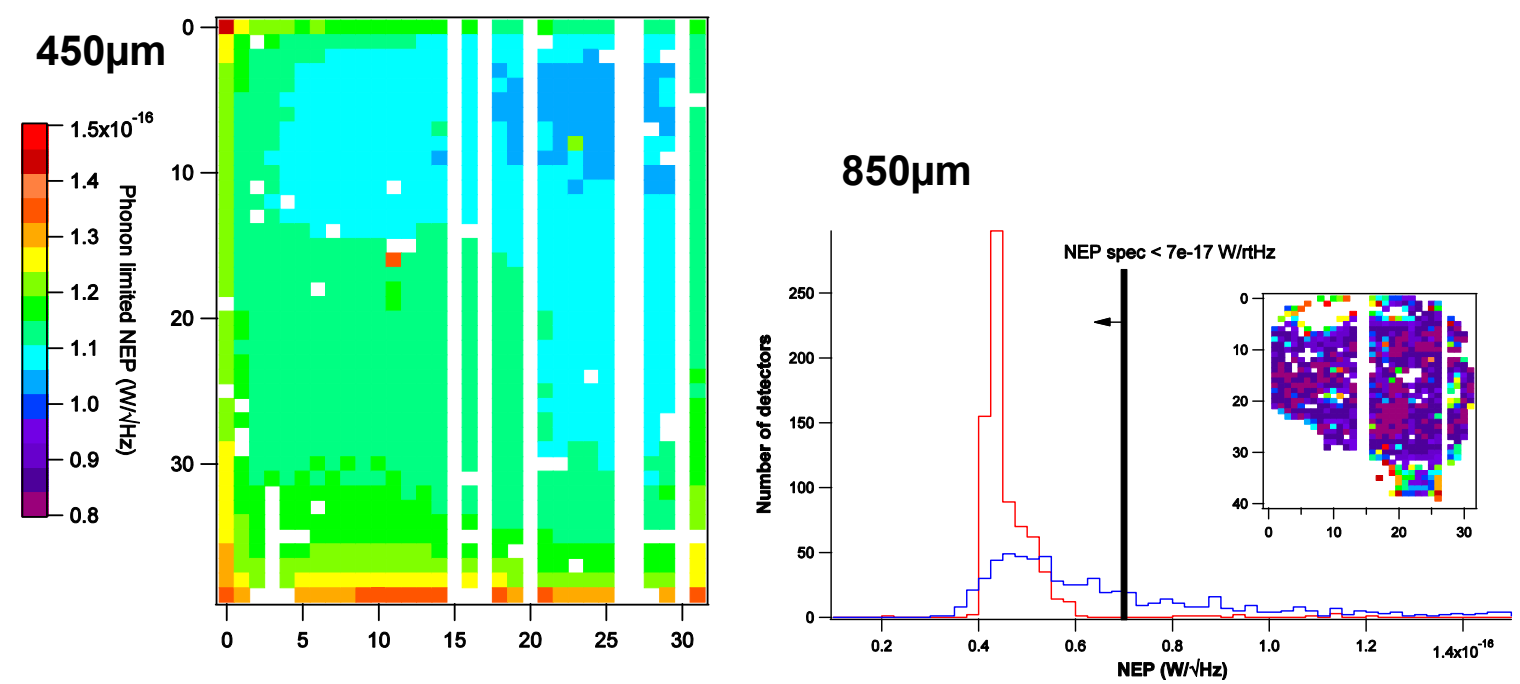

Figure 8. (Left) Phonon noise limited NEP for the 450um science grade detectors. (Right) Histogram showing the $850 \mu \mathrm{m}$ phonon noise limited NEP (red) and a typical measured (from the current noise) NEP (blue) at the start of Science Observing. The insert shows the $850 \mu \mathrm{m}$ measured NEP detector map.

The $450 \mu \mathrm{m}$ science grade sub-array achieves the goal to have the intrinsic detector NEP at least a factor of two less than the background from the sky and telescope: 992 detectors (78\%) have measured NEP less than $1.2 \mathrm{e}-16 \mathrm{~W} / \mathrm{rtHz}$. For the $850 \mu \mathrm{m}$ science grade sub-array, 703 detectors $(55 \%)$ meet the more stringent requirements. However in practice (see Figure 8) the variation of Tc (particularly for the 850), compromises in the SQUID setup and external sources of noise picked up by the TES influence the array performance tremendously.
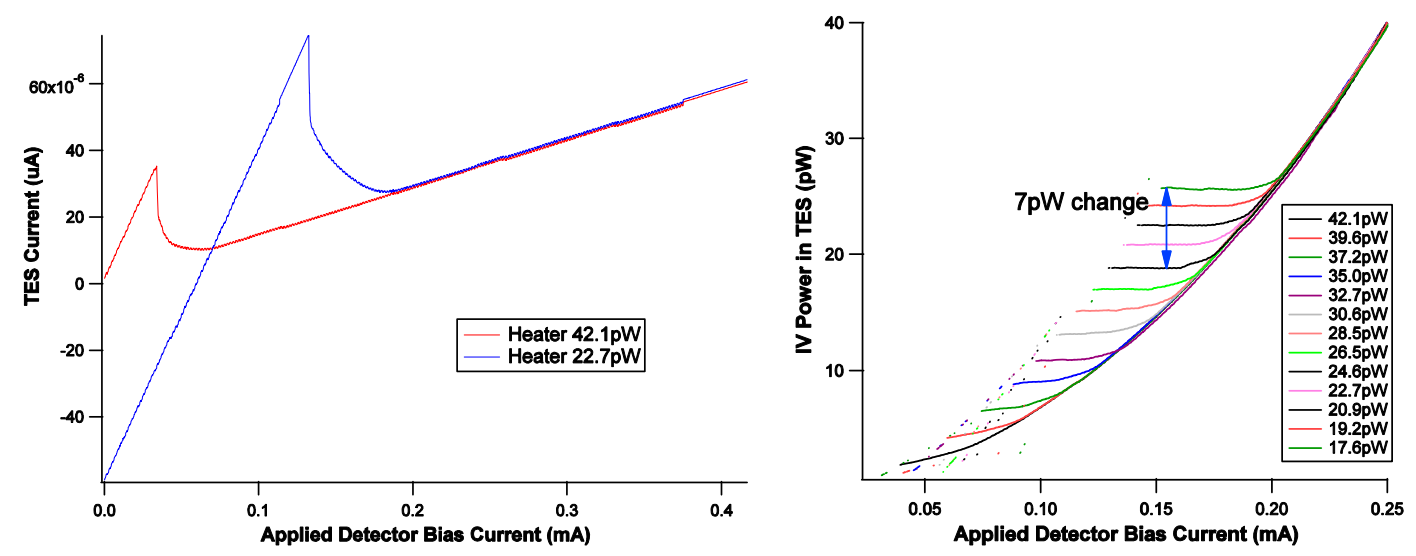

Figure 9. Current-voltage (IV) characteristics for a typical $850 \mu \mathrm{m}$ detector, at two heater powers are plotted. (Right) IV power is plotted against applied detector bias for a range of heater settings.

IV characteristics for a typical $850 \mu \mathrm{mTES}$ at different heater powers are shown in figure 9 . From the IV curves and by assuming that the shunt resistance is $5 \mathrm{~m} \Omega$ (the design value), the TES resistance and IV power in the superconducting 
transition can be calculated. An (almost) constant power plateau in the transition is observed in Figure 9. The effective heater resistance per detector and any variation across the array can be calculated from IV and power curves for different heater settings.

The normal state resistance of the $850 \mu \mathrm{m}$ detectors on the science grade array are less than $40 \%$ of the expected values (see Fig 10), compared to the commissioning grade $850 \mu \mathrm{m}$ the $450 \mu \mathrm{m}$ science grade array. The total power handling and intrinsic NEP of the science grade 850 array are both to specification. The impact of the reduced normal state resistance is to narrow the width of the transition in terms of applied detector bias for each detector. This compounds the problem of the variation in transition temperatures across the array and makes it harder to setup the whole sub-array into transition with a single applied bias and heater setting. The reduced detector resistances values maybe also push some of the detectors into instability, depending upon the effective time constant of the TES and the strength of any electro thermal feedback.
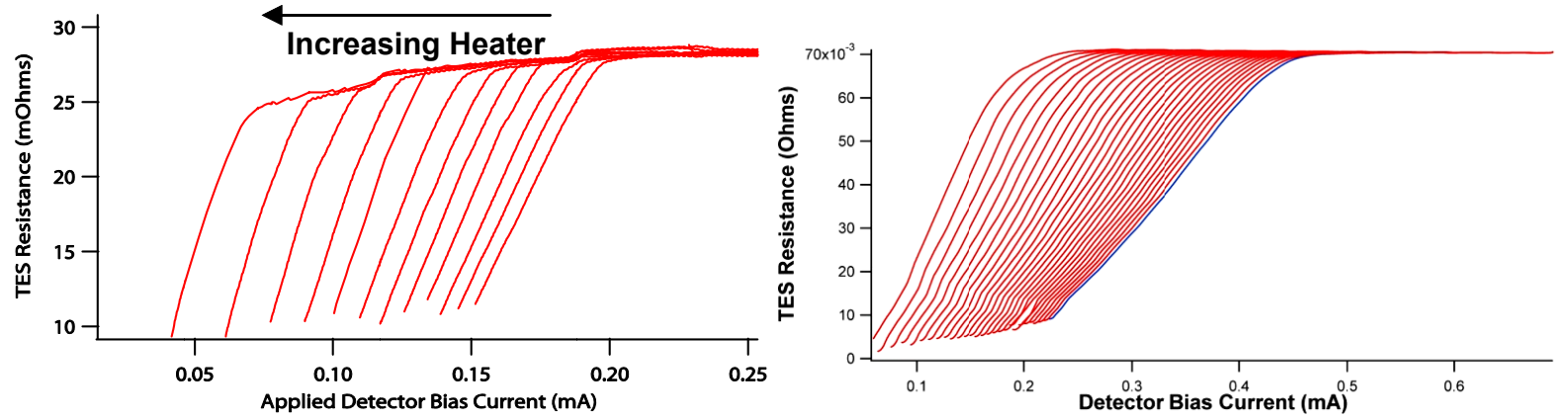

Figure 10. The electrical resistance of an $850 \mu \mathrm{m}$ detector is plotted against applied detector bias current, for a series of heater powers. The TES resistance is constant in the normal state. (Right) A similar plot is shown the commissioning grade $850 \mu \mathrm{m}$ sub-array.

The small signal theory for voltage biased TES predict a simple relation between the bias voltage and the dc current responsivity in the transition: $s=-1 / V$.

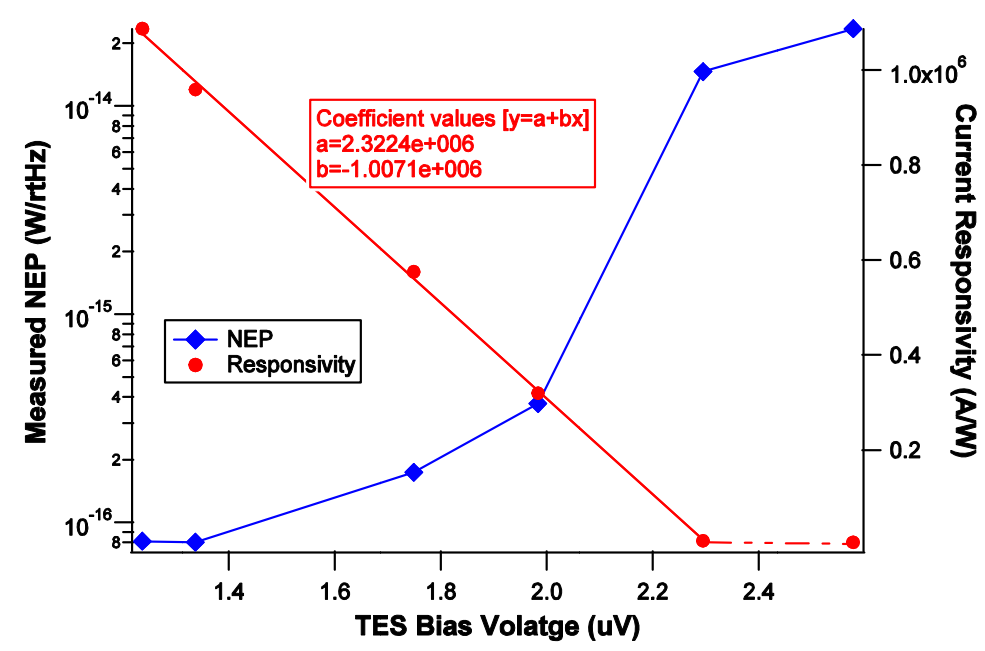

Figure 11. NEP (blue) and current responsivity (red) plotted against the applied TES bias voltage, with the heater power constant. The solid red line is a fit to the current responsivity, for bias voltages $<2.3 \mu \mathrm{V}$.

For a fixed heater power we measured the relation between the TES bias voltage and the current responsivity and also the bias and the electrical NEP. Figure 10 illustrates these relations for a typical detector on the $850 \mu \mathrm{m}$ commissioning grade array. We observe similar results with the science grade arrays. The linear fit of the responsivity against bias voltage over the bias voltage range $<2.3 \mu \mathrm{V}$, agrees well with theory. 
The measured NEP (from the mean detector current noise between 2 to $10 \mathrm{~Hz}$ ) starts to increase (degrade) as the responsivity falls below $1 \times 10^{6} \mathrm{~A} / \mathrm{W}$. At lower bias voltages the detector became unstable and above $2.3 \mu \mathrm{V}$ the detector is becoming mostly normal (ignoring hysteresis). One explanation for the rise in NEP as the responsivity reduces is the increasing dominance of noise from the SQUID multiplexer, which is higher on the commissioning grade array during this measurement.

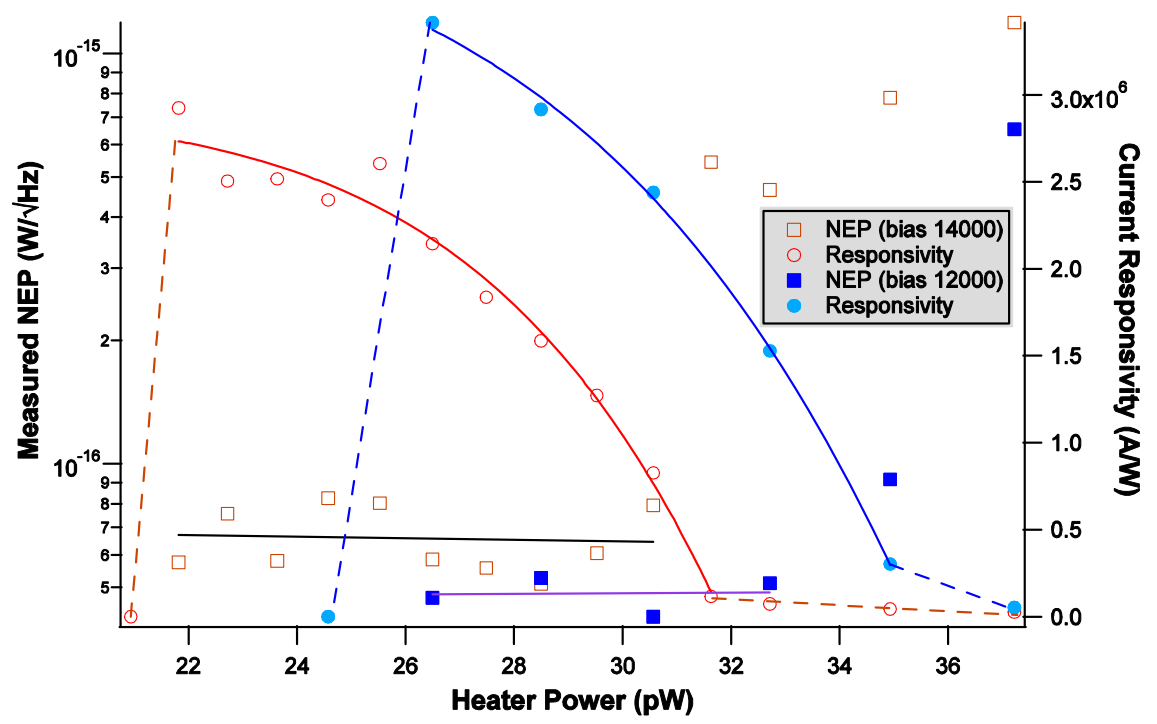

Figure 12. Current responsivity and NEP are plotted against heater power, for a fixed detector bias. Two detector biases are shown. The solid lines are fits to the data. (The measurements are for a typical detector on the $850 \mu \mathrm{m}$ science grade sub-array).

For a fixed detector bias and varying the heater, we found that the responsivity increases with reduced heater power. The NEP is broadly constant (once the responsivity is $>1 \times 10^{6} \mathrm{~A} / \mathrm{W}$ ) to lower heater power, despite the increasing responsivity. For the science grade $850 \mu \mathrm{m}$ detector (see Figure 12), a lower detector bias resulted in a higher responsivity, but also a lower constant NEP. As the heater is reduced further the responsivity suddenly collapses as the TES goes superconducting.

One aspect that is worth noting from this figure: We show that the responsivity varies with power loading on the TES. This will include optical power. Therefore observing bright calibrator sources for example, such as Mars, may introduce a significant nonlinear response; similarly if the varying sky background is not corrected sufficiently or often enough with the heater, the responsivity may change during an observation.

\subsection{Setup for optical testing and on-sky calibration}

To optimise the arrays for on-sky calibration and science observing the TES bias and heater settings are chosen to maximise the number of detectors that meet or exceed the NEP requirements. However out of spec detectors also contribute to the final maps. Therefore a more sophisticated optimisation is to weight all the working (i.e. in transition) detectors based on their NEP and optimise the TES bias and heater to maximise the mapping speed of the instrument.

Data from unstable or variable detectors are thrown out by the data reduction pipeline but it was found best to turn these detectors off in advance via the setup, as flux jumps and out of lock elements with rapidly ramping SQ1 feedback can cause steps and increased noise on adjacent detectors. The optimised array setups for observing typically return $800-$ 900 working (as determined by a linear response to a ramped signal on the heater, used to calibrate or flatfield) detectors at each wavelength with a spread or distribution of NEP as shown Figure 9 for the $850 \mu \mathrm{m}$ sub-array.

The planned upgrade to the CEM is expected to increase the number and quality of working TES, particularly on the $850 \mu \mathrm{m}$ array. The effect of temperature oscillations in the dilution fridge on the output of the detectors is also expected to diminish. The $1 \mathrm{~K}$ box is cooled directly by the dilution fridge still. The fridge oscillations are largest in the still and therefore are adversely coupled to the detector arrays via the CEM. 
The low frequency oscillations from the fridge as seen by the detectors, present a challenge to the data reduction team in making flat maps. In Figure 13 we show a typical time stream and spectrum for an $850 \mu \mathrm{m}$ TES in the dark. The large fridge oscillation has a period of approximately 30 seconds. Also visible in the spectrum is a thermal pickup from the pulse tube coolers ( $1.5 \mathrm{~Hz}$ plus higher harmonics).

The frequency spectrums are generally flat from about $1 \mathrm{~Hz}$ until the roll off due to the MCE filter $(70 \mathrm{~Hz})$. However the low frequency pickup (from the fridge) and 1/f from the MUX and detectors, together with any discrete features in the frequency spectrum constrain the observing modes for the instrument.
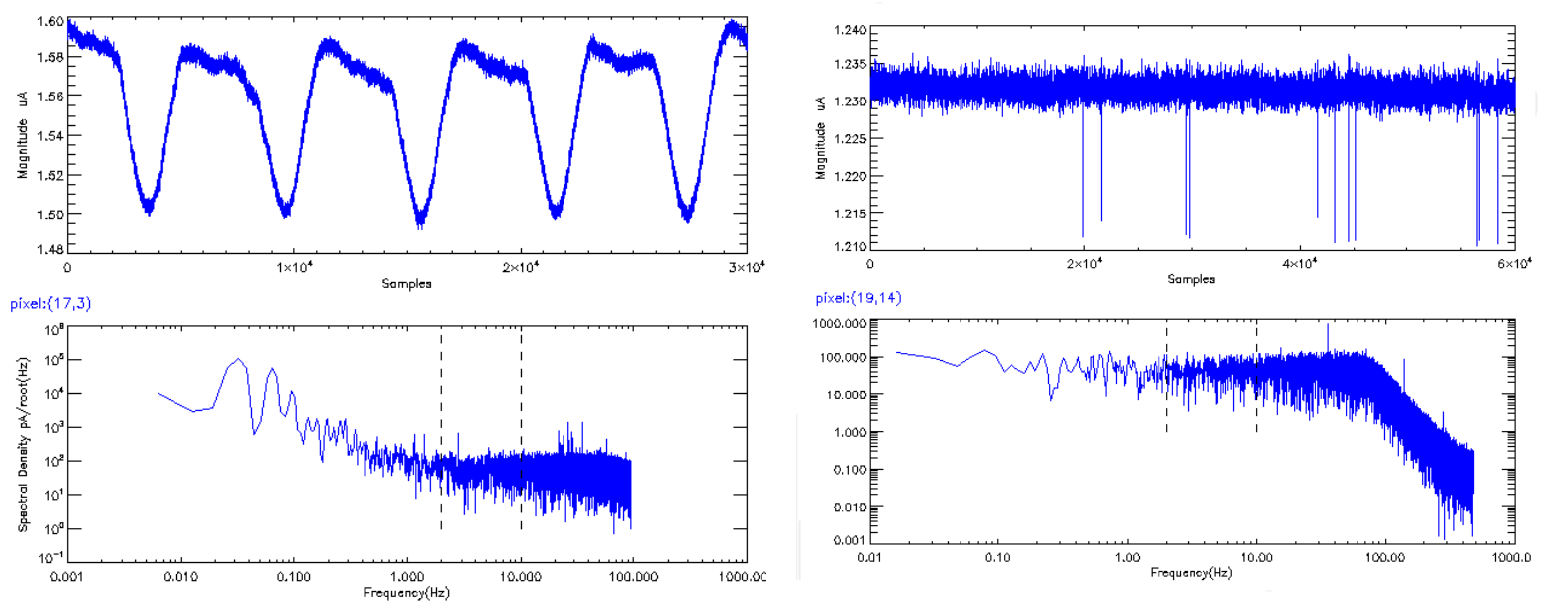

Figure 13. (Left) A typical time stream and spectrum from an $850 \mu \mathrm{m}$ detector in the dark, showing periodic fridge oscillations. (Right) With the detector in the normal state and the output sampled at a higher frame rate $(1 \mathrm{kHz})$, to show the MCE filter characteristics.

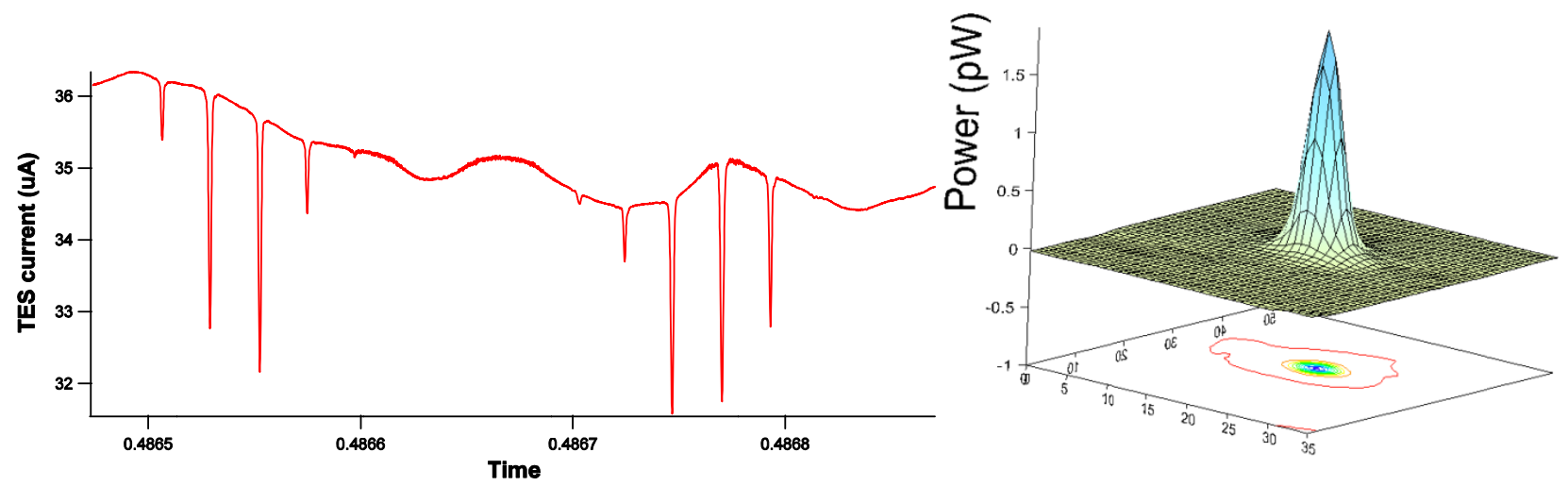

Figure 14. Detector current for a single $850 \mu \mathrm{m}$ TES, scanning over Mars. (Right) The resultant scan map of Mars at $850 \mu \mathrm{m}$, for a 60 second observation. The amplitudes of the signal are in units of power $(\mathrm{pW})$.

A short section of a quick scan observation of Mars, output from a single TES at $850 \mu \mathrm{m}$, is shown in Figure 14 . This observation highlights the effect of the fridge oscillations on the science data. The mars signals are the large downward 'spikes' in the detector current. For much less bright sources the fridge temperature pickup dwarfs the signal from the source. One of the challenges for the data reduction is to remove this background while preserving flux from faint extended sources. As we show in reference [2]; we are making progress in this respect. 


\section{CONCLUSION}

We have described the characterisation of the first science grade arrays for SCUBA-2. The intrinsic properties of the TES arrays at both wavelengths achieve the design requirements and goals for the instrument, with much higher detector 'pixel' yields than we achieved on prototype or commissioning grade arrays.

However the performance of the science grade arrays in the instrument has been hampered by a number of issues which are principally thermal in nature. We expect an improvement in performance with the new cold electronics modules that will be installed as part of the array upgrade this summer.

The optical performance of the detector arrays and the results of on-sky calibration are discussed separately.

\section{REFERENCES}

[1] Craig, S. et al., "SCUBA-2: engineering and commissioning challenges of the world largest sub-mm instrument at the JCMT", Proc. SPIE 7741, (2010).

[2] Holland, W. S. et al., "SCUBA-2: first results and on-sky performance", Proc. SPIE 7741, (2010).

[3] Dempsey, J. T. et al., "Extinction correction and on-sky calibration of SCUBA-2", Proc. SPIE 7741, (2010).

[4] Audley, M. D., Duncan, W. D., Holland, W. S. et al., "Fabrication of the SCUBA-2 detector arrays", Nuclear Instruments and Methods in Physics Research A 520, 483-486 (2004).

[5] deKorte, P.A.J., Beyer, J., Deiker, S., Hilton, G.C., Irwin, K.D., et al., "Time-division superconducting quantum interference device multiplexer for transition-edge sensors", Rev. Sci. Instrum. 74, 3807-3815 (2003)

[6] Irwin. K.D., "An application of electrothermal feedback for high resolution cryogenic particle detection", Appl. Phys. Lett., 66(15), 1998-2000 (1995).

[7] Irwin K. D. and Hilton G. C., [Cryogenic Particle Detection], C. Enss (Ed.) Topics Appl. Phys. 99, Springer-Verlag Berlin Heidelberg, 63-149 (2005).

[8] Woodcraft, A. L., Ade, P.A.R., Bintley, D. et al., "Electrical and optical measurements on the first SCUBA-2 prototype 1280 pixel submillimetre superconducting bolometer array", Review of Scientific Instruments, 78, 024502 (2007).

[9] Gao, X., Kelly, D. et al., “Automatic setup of SCUBA-2 detector arrays”, Proc. SPIE, Vol. 7020, 702025 (2008)

[10] Battistelli, E. S., Amiri, M., Burger, B. et al., "Functional Description of Read-out Electronics for Time-Domain Multiplexed Bolometers for Millimeter and Sub-millimeter Astronomy", Journal of Low Temperature Physics, 151, 908914 (2008). 